\title{
MMSE Filtering Performance of Dual-Hop Amplify-and-Forward Multiple-Access Channels
}

\author{
Symeon Chatzinotas, Member, IEEE
}

\begin{abstract}
Relay channels have been heavily studied during the last years as a means of improving spectral efficiency, availability and coverage in combination with multiple antenna transceivers. Relaying systems can comprise many hops but the most practical approach for the time being would be a dual-hop system. In addition, the simplest method of relaying in terms of transceiver complexity is amplify and forward. In this context, we investigate the MMSE filtering performance of a dual-hop amplify-andforward channel MIMO multiple-access channel and we propose a tight lower bound for the average MMSE based on the principles of free probability theory. The accuracy of the derived closed-form expressions and the effect of the normalized system parameters, such as first/second hop power/load, are evaluated through numerical results. It is established that the performance of MMSE receiver greatly depends on the first hop power, while increasing the second hop power marginally improves the performance. Furthermore, inequality conditions are expressed for first/second hop load in order to achieve low average MMSE.
\end{abstract}

Index Terms-Amplify and Forward Relaying, Multiple Access Channel, Minimum Mean Square Error, Large System Analysis.

\section{INTRODUCTION}

The Dual-Hop (DH) Amplify-and-Forward (AF) relay channel has attracted a great deal of attention mainly due to its low complexity and its manyfold benefits, such as coverage extension and decreased outage probability. In addition, the MIMO Multiple Access Channel (MAC) has been studied heavily during the last years since it comprises a fundamental channel model for multiuser uplink cellular [1] and multibeam return link [2] communications. In this paper, we combine these two research areas by considering a MIMO MAC where each user has to go through an array of AF relays in order to reach the receiver. We consider joint Minimum Mean Square Error (MMSE) filtering followed by single user decoding and the figure of merit under investigation is the average MMSE.

In existing literature, the ergodic capacity assuming Rayleigh channel has been studied in the large system through Stieltjes' fixed-point equations [3] and through replica analysis [4]. Furthermore, authors in [5] have considered AF in a cellular scenario with Base Station cooperation and approached the problem using replica analysis for determining the ergodic sum-rate for optimal and MMSE receivers. However, most of the aforementioned results focus on ergodic capacity [6], while

S. Chatzinotas is with the Interdisciplinary Centre for Security, Reliability and Trust, University of Luxembourg (http://www.securityandtrust.lu) e-mail: Symeon.Chatzinotas@uni.lu. the solution is provided in the form of fixed-point equations containing functionals of correlation and power coefficients. In this paper, we propose a a tight lower bound for the average MMSE following a free-probabilistic approach which can provide solutions by solving a quartic polynomial. This could be also seen as an extension of the work in [7] about double-scattering MIMO channels. It should be noted that the ergodic capacity can be also calculated by solving a quartic polynomial as shown in [8].

The remainder of this paper is structured as follows: Section II introduces the system model and provides a review of related work. Section III describes the free probability derivations and the main MMSE results. Section IV verifies the accuracy of the analysis by comparing with Monte Carlo simulations and evaluates the effect of the channel parameters. Section V concludes the paper.

\section{SySTEM MOdEL}

The considered system model includes multiple singleantenna users $(M)$, multiple single-antenna relay stations $(K)$ and a single multi-antenna $(N)$ receiver $^{1}$. It should be noted that relay stations cannot cooperate and thus precoding optimization [9] is not possible. It is assumed that the relays are employed for coverage extension and thus the direct link between users and receiver is negligible and can be ignored. Gaussian input is considered at the user-side, while neither users nor relays are aware of the Channel State Information (CSI). The received signal in vectorial form can be expressed as follows:

$$
\begin{aligned}
& \mathbf{y}_{1}=\mathbf{H}_{1} \sqrt{\mu} \mathbf{x}_{1}+\mathbf{z}_{1} \text { and } \mathbf{y}_{2}=\mathbf{H}_{2} \sqrt{\nu} \mathbf{y}_{1}+\mathbf{z}_{2} \Leftrightarrow \\
& \mathbf{y}_{2}=\mathbf{H}_{2} \mathbf{H}_{1} \sqrt{\mu \nu} \mathbf{x}_{1}+\mathbf{H}_{2} \sqrt{\nu} \mathbf{z}_{1}+\mathbf{z}_{2},
\end{aligned}
$$

where the $M \times 1$ vector $\mathbf{x}_{1}$ denotes the user transmitted symbol vector with $\mathbb{E}\left[\mathbf{x}_{1} \mathbf{x}_{1}^{H}\right]=\mathbf{I}, \mathbf{y}_{1}$ denotes the $K \times 1$ received symbol vector by the relays and the $K \times 1$ vector $\mathbf{z}_{1}$ denotes AWGN at relay-side with $\mathbb{E}\left[\mathbf{z}_{1}\right]=\mathbf{0}$ and $\mathbb{E}\left[\mathbf{z}_{1} \mathbf{z}_{1}^{H}\right]=\mathbf{I}$. The received signal $\mathbf{y}_{1}$ is amplified and forwarded and as a result $\mathbf{y}_{2}$ denotes the $N \times 1$ received symbol vector by the receiver and the $N \times 1$ vector $\mathbf{z}_{2}$ denotes AWGN at receiver-side with $\mathbb{E}\left[\mathbf{z}_{2}\right]=\mathbf{0}$ and $\mathbb{E}\left[\mathbf{z}_{2} \mathbf{z}_{2}^{H}\right]=\mathbf{I}$. The available power per users is denoted by $\mu$, while $\nu$ represents the amplification power per relay. Finally, $\mathbf{H}_{1}, \mathbf{H}_{2}$ include zero-mean independent

\footnotetext{
${ }^{1}$ However, the presented analysis is straightforwardly applicable to multiantenna users and relays under uniform power allocation.
} 
identically distributed (i.i.d.) complex circularly symmetric (c.c.s.) elements modelling fast fading.

The optimal SIC receiver involves successive interference cancellation [10] which is highly complex and can suffer from error propagation. In this direction, we consider a less complex receiver which involves Mimimum Mean Square Error filtering (MMSE) followed by single-user decoding. Since, this is a linear operation on the received vector $\mathbf{y}_{2}$, we assume that $N \geq M$, namely the number of receive antennas is at least as large as the number of served users. The performance of the MMSE receiver is dependent on the achieved MMSE averaged over users and channel realizations and is given by [11]:

$$
\begin{aligned}
& \text { mmse }_{\text {avg }}=\mathbb{E}_{\mathbf{H}_{1}, \mathbf{H}_{2}}\left[\frac{1}{M} \sum_{m=1}^{M} \text { mmse }_{m}\right] \\
& =\mathbb{E}\left[\frac{1}{M} \sum_{m=1}^{M}\left[\left(\mathbf{I}+\mu \nu \mathbf{H}_{1}^{H} \mathbf{H}_{2}^{H}\left(\mathbf{I}+\nu \mathbf{H}_{2} \mathbf{H}_{2}^{H}\right)^{-1} \mathbf{H}_{2} \mathbf{H}_{1}\right)^{-1}\right]_{m, m}\right] \\
& =\mathbb{E}\left[\frac{1}{M} \operatorname{tr}\left\{\left(\mathbf{I}+\nu \mathbf{H}_{2}\left(\mathbf{I}+\mu \mathbf{H}_{1} \mathbf{H}_{1}^{H}\right) \mathbf{H}_{2}^{H}\right)^{-1}\left(\mathbf{I}+\nu \mathbf{H}_{2} \mathbf{H}_{2}^{H}\right)\right\}\right] .
\end{aligned}
$$

To simplify notations during mathematical analysis, the following auxiliary variables are defined:

$$
\begin{aligned}
\mathbf{M} & =\mathbf{I}+\mu \mathbf{H}_{1} \mathbf{H}_{1}^{H}, \quad \tilde{\mathbf{M}}=\mathbf{I}+\nu \mathbf{H}_{2} \mathbf{H}_{2}^{H} \\
\mathbf{N} & =\mathbf{H}_{1} \mathbf{H}_{1}^{H}, \tilde{\mathbf{N}}=\mathbf{H}_{2}^{H} \mathbf{H}_{2} \\
\mathbf{K} & =\mathbf{H}_{2}^{H} \mathbf{H}_{2}\left(\mathbf{I}+\mu \mathbf{H}_{1} \mathbf{H}_{1}^{H}\right)=\tilde{\mathbf{N}} \mathbf{M} \\
\tilde{\mathbf{K}} & =\mathbf{H}_{2}\left(\mathbf{I}+\mu \mathbf{H}_{1} \mathbf{H}_{1}^{H}\right) \mathbf{H}_{2}^{H} \\
\beta & =M / K, \gamma=K / N
\end{aligned}
$$

where $\beta, \gamma$ are the ratios of horizontal to vertical dimensions of matrix $\mathbf{H}_{1}, \mathbf{H}_{2}^{H}$ with $\beta \gamma \leq 1$. It should be noted that the values $\beta$ and $\gamma$ characterize the first hop $(F H L)$ and second hop load (SHL) respectively. In other words, FHL corresponds to users per relay, while SHL corresponds to relays per receive antenna.

\section{iII. Free Probabilistic Analysis \& Main Results}

In order to calculate the system performance analytically, we resort to asymptotic analysis which entails that the dimensions of the channel matrices grow to infinity assuming proper normalizations. It has already been shown in many occasions that asymptotic analysis yields results which are also valid for finite dimensions [12]-[14]. In other words, the expressions of interest converge quickly to a deterministic value as the number of channel matrix dimensions increases. It should be noted that while the channel dimensions $K, M, N$ grow large the loads $\beta, \gamma$ are kept constant.

Using asymptotic analysis, the average MMSE can be expressed as:

$$
\begin{aligned}
\text { mmse }_{\text {avg }} & =\lim _{K, M, N \rightarrow \infty} \mathbb{E}_{\tilde{\mathbf{K}}, \tilde{\mathbf{M}}}\left[\frac{1}{M} \operatorname{tr}\left\{(\mathbf{I}+\nu \tilde{\mathbf{K}})^{-1} \tilde{\mathbf{M}}\right\}\right] \\
& \stackrel{(a)}{\geq} \lim _{K, M, N \rightarrow \infty} \mathbb{E}_{\tilde{\mathbf{K}}, \tilde{\mathbf{M}}}\left[\frac{1}{M} \sum_{m=1}^{M} \frac{\lambda_{M-m+1}(\tilde{\mathbf{M}})}{1+\nu \lambda_{m}(\tilde{\mathbf{K}})}\right]
\end{aligned}
$$

$$
\rightarrow \int_{0}^{1} \frac{F_{\tilde{\mathbf{M}}}^{-1}(1-x)}{1+\nu F_{\tilde{\mathbf{K}}}^{-1}(x)} \mathrm{d} x
$$

where step (a) follows from [15] and $F_{\mathbf{X}}^{-1}$ denotes the inverse function of the asymptotic eigenvalue cumulative density function (a.e.c.d.f.). In the asymptotic regime, the ordered eigenvalues can be represented by a continuous deterministic function given by the inverse function of the a.e.c.d.f. [2], also known as inverse transform sampling. To calculate the expression of eq. (5), it suffices to derive the asymptotic densities of $\tilde{\mathbf{K}}, \tilde{\mathbf{M}}$, which can be achieved through the principles of free probability theory [16]-[19] as described in the following paragraphs.

\section{A. Free Probability Results}

Free probability (FP) has been proposed by Voiculescu [16] and has found numerous applications in the field of wireless communications. More specifically, FP has been applied for capacity derivations of variance profiled [20], correlated [21] Rayleigh channels, as well as Rayleigh product channels [7]. Furthermore, it has been used for studying cooperative relays [22], interference channels [23] and interference alignment scenarios [24]. The advantage of FP methodology compared to other techniques, such as Stieltjes method, replica analysis and deterministic equivalents, is that the derived formulas usually require just a polynomial solution instead of fixedpoint equations. However, the condition for these simpler solutions is that the original asymptotic eigenvalue probability density functions (a.e.p.d.f.) of the involved channel matrices can be expressed in polynomial form [25]. longequation

Definition 3.1: Considering a Gaussian $K \times N$ channel matrix $\mathbf{H} \sim \mathcal{C N}(\mathbf{0}, \mathbf{I} / K)$, the a.e.p.d.f. of $\mathbf{H H}^{H}$ converges a.s. to the non-random limiting eigenvalue distribution of the Marčenko-Pastur law [26], whose density functions are given by

$$
\begin{aligned}
f_{\mathbf{H H}^{H}}^{\infty}(x) & \rightarrow f_{\mathrm{MP}}(x, y) \\
f_{\mathrm{MP}}(x, y) & =(1-y)^{+} \delta(x)+\frac{\sqrt{(x-a)^{+}(b-x)^{+}}}{2 \pi x}
\end{aligned}
$$

where $a=(1-\sqrt{y})^{2}, b=(1+\sqrt{y})^{2}$ and $\eta$-transform, $\Sigma$ transform are given by [13]

$$
\begin{gathered}
\eta_{\mathbf{H H}^{H}}(x, y)=1-\frac{\phi(x, y)}{4 x} \\
\phi(x, y)=\left(\sqrt{x(1+\sqrt{y})^{2}+1}-\sqrt{x(1-\sqrt{y})^{2}+1}\right)^{2} \\
\Sigma_{\mathbf{H H}^{H}}(x, y)=\frac{1}{y+x} \text { and } \Sigma_{\mathbf{H}^{H} \mathbf{H}}(x, y)=\frac{1}{1+y x}
\end{gathered}
$$

and $y=N / K$ is the ratio of the horizontal to the vertical dimension of the $\mathbf{H}$ matrix.

Let us assume that $\mathbf{H}_{1} \sim \mathcal{C N}(\mathbf{0}, \mathbf{I} / K)$ and $\mathbf{H}_{2} \sim$ $\mathcal{C N}(\mathbf{0}, \mathbf{I} / N)^{2}$.

Theorem 3.1: The a.e.c.d.f. of $\mathbf{N}$ is given by:

\footnotetext{
${ }^{2}$ The method described herein could be extended for variance-profiled Gaussian matrices using the approximation in [20].
} 


$$
\eta_{\mathbf{M}}^{-1}(x, \mu, \beta)=\frac{-x \mu-\beta \mu+\mu-1+\sqrt{x^{2} \mu^{2}+2 x \tilde{\mu}^{2} \beta-2 x \tilde{\mu}^{2}-2 x \mu+\beta^{2} \mu^{2}-2 \beta \mu^{2}+2 \beta \mu+\mu^{2}+2 \mu+1}}{2 x \mu}
$$

$$
\begin{gathered}
F_{\mathbf{N}}(x)=\frac{1}{2}+\frac{1-\beta}{2 \pi} \arcsin \left(\frac{\beta+1}{2 \sqrt{\beta}}-\frac{(1-\beta)^{2}}{2 x \sqrt{\beta}}\right) \\
+x f_{\mathrm{MP}}(x, \beta)-\frac{\beta+1}{2 \pi} \arcsin \left(\frac{\beta+1}{2 \sqrt{\beta}}-\frac{x}{2 \sqrt{\beta}}\right) .
\end{gathered}
$$

Proof: The c.d.f. follows from eq.(7) after integration as in $[27]^{3}$.

Theorem 3.2: The a.e.c.d.f. of $\mathbf{M}$ is given by:

$$
\begin{aligned}
& F_{\mathbf{M}}(x)=\frac{1}{2}-\frac{1-\beta}{2 \pi} \arcsin \left(-\frac{\beta+1}{2 \sqrt{\beta}}+\frac{(1-\beta)^{2} \mu}{2(x-1) \sqrt{\beta}}\right) \\
+ & x f_{\mathbf{M}}\left(\frac{x-1}{\mu}, \beta, \mu\right)-\frac{\beta+1}{2 \pi} \arcsin \left(\frac{\beta+1}{2 \sqrt{\beta}}-\frac{x-1}{2 \mu \sqrt{\beta}}\right) .
\end{aligned}
$$

Similarly, the a.e.c.d.f. of $\tilde{\mathbf{M}}$ is given by substituting $\mu=\nu$ and $\beta=\gamma$.

Proof: Since a monotonic function is used for the change of variable, the c.d.f. follows from $F_{\mathbf{M}}(x)=F_{\mathbf{N}}\left(\frac{x-1}{\mu}\right)$.

Lemma 3.1: The a.e.p.d.f. of $\mathbf{M}$ converges a.s. to:

$f_{\mathrm{M}}^{\infty}(x, \mu, \beta) \rightarrow \frac{\sqrt{(x-1-\mu+2 \mu \sqrt{\beta}-\mu \beta)(\mu+2 \mu \sqrt{\beta}+\mu \beta-x+1)}}{2 \mu \pi(x-1)}$.

Proof: The a.e.p.d.f. can be calculated considering the chain rule through the transformation $z(x)=(1+\mu x)$, where $z$ and $x$ represent the eigenvalues of $\mathbf{M}$ and $\mathbf{H} \mathbf{H}^{H}$ respectively:

$$
f_{\mathbf{M}}^{\infty}(x)=\left|\frac{1}{z^{\prime}\left(z^{-1}(x)\right)}\right| \cdot f_{\mathbf{H H}^{H}}^{\infty}\left(z^{-1}(x)\right)=\frac{1}{\mu} f_{\mathbf{H H}^{H}}\left(\frac{x-1}{\mu}\right) .
$$

Theorem 3.3: The inverse $\eta$-transform of $\mathbf{M}$ is given by (6) at the top of the page.

Proof: Due to lack of space only an outline of the proof is provided. The proof is based on the definition of $\eta$-transform [13] using Cauchy integration after appropriate change of variables as in [23].

Theorem 3.4: The inverse $\eta$-transform of $\mathbf{K}$ is given by:

$$
\eta_{\mathbf{K}}^{-1}(x)=\frac{1}{1+\gamma x-\gamma} \eta_{\mathbf{M}}^{-1}(x)
$$

Proof: Given the asymptotic freeness between unitarily invariant matrices $\tilde{\mathbf{N}}$ and $\mathbf{M}$, the $\Sigma$-transform of $\mathbf{K}$ is given by multiplicative free convolution:

$$
\begin{aligned}
\Sigma_{\mathbf{K}}(x) & =\Sigma_{\tilde{\mathbf{N}}}(x) \Sigma_{\mathbf{M}}(x) \stackrel{(a)}{\Longleftrightarrow} \\
\left(-\frac{x+1}{x}\right) \eta_{\mathbf{K}}^{-1}(x+1) & =\frac{1}{1+\gamma x}\left(-\frac{x+1}{x}\right) \eta_{\mathbf{M}}^{-1}(x+1),
\end{aligned}
$$

where step $(a)$ combines $\Sigma$-transform definition [13] and eq. (10). The variable substitution $y=x+1$ yields eq. (15).

Theorem 3.5: The Stieltjes transform of $\mathbf{K}$ is given by the solution of the quartic polynomial:

\footnotetext{
${ }^{3}$ It should be noted that the expression in [27, Eq.(4)] contains an error in the second term.
}

$$
\begin{array}{r}
2 \mu^{2} x^{2} \mathcal{S}_{\mathbf{K}}(x)^{4} \\
+\left(2 \mu^{2} x^{2}+4(1-\beta) \mu^{2} x\right) \mathcal{S}_{\mathbf{K}}(x)^{3} \\
+\left(2\left(\left(2-\beta-\gamma^{-1}\right) \mu^{2}-\mu\right) x+2(\beta-1)^{2} \mu^{2}\right) \mathcal{S}_{\mathbf{K}}(x)^{2} \\
+\left(2\left(1-\gamma^{-1}\right) \mu^{2} x+2(\beta-1)\left(\gamma^{-1}-1\right) \mu^{2}+2(\beta-1) \mu\right) \mathcal{S}_{\mathbf{K}}(x) \\
+\left(\gamma^{-1}-1\right)^{2} \mu^{2}+2 \gamma^{-1} \mu+1
\end{array}
$$

Proof: The Stieltjes-transform of a positive semidefinite matrix $\mathbf{K}$ can be derived by its $\eta$-transform using

$$
\mathcal{S}_{\mathbf{K}}(x)=-\frac{\eta_{\mathbf{K}}(-1 / x)}{x} .
$$

Applying suitable change of variables yields:

$$
x \eta_{\mathbf{K}}^{-1}\left(-x \mathcal{S}_{\mathbf{K}}(x)\right)+1=0 .
$$

Inserting eq. (6) completes the proof after some algebraic simplifications.

Lemma 3.2: The a.e.p.d.f. of $\mathbf{K}$ is obtained by determining the imaginary part of the Stieltjes transform $\mathcal{S}$ for real arguments:

$$
f_{\mathbf{K}}^{\infty}(x)=\lim _{y \rightarrow 0^{+}} \frac{1}{\pi} \mathfrak{I}\left\{\mathcal{S}_{\mathbf{K}}(x+\mathrm{j} y)\right\}
$$

and the a.e.p.d.f. of $\tilde{\mathbf{K}}$ is given by:

$$
f_{\tilde{\mathbf{K}}}^{\infty}(x)=\left(1-\frac{1}{\gamma}\right)^{+} \delta(x)+\frac{1}{\gamma} f_{\mathbf{K}}^{\infty}(x) .
$$

Remark 3.1: The average MMSE mmse ${ }_{\text {avg }}$ is given by eq. (5) where $F_{\tilde{\mathrm{M}}}^{-1}(x)$ can be calculated using inversion over the a.e.c.d.f. in Theorem 3.2 and $F_{\tilde{\mathbf{K}}}^{-1}(x)$ using numerical integration and inversion over the a.e.p.d.f. in lem. 3.2.

\section{Numerical Results}

In order to verify the accuracy of the derived expressions and gain insights on the system performance of the considered model, some numerical results are presented in this section. In the context of these simulations, the Gaussian matrices $\mathbf{H}_{1}, \mathbf{H}_{2}$ are randomly generated for $10^{3}$ fading instances and the resulting capacity is calculated by averaging according to eq. (2). Figure 1 depicts the effect of power levels $(\mu, \nu)$ on the average MMSE for $\beta=\gamma=1$. The upper mesh plot was calculated through Monte Carlo simulations of eq. (2), whereas the lower mesh plot represents the bound which was calculated using Remark 3.1. It can be seen that the proposed bound is tight for all values of $\mu, \nu$. It should be noted that the average MMSE decreases substantially with $\mu$, while it slowly decreases with $\nu$. Subsequently, Figure 2 depicts the effect of number of relays $K$ on the average MMSE while keeping a constant number or users and receive antennas $M=N=10$. Solid curves are plotted analytically, while points are calculated based on Monte Carlo simulations. It can be seen that when the number of relays is lower than the number of users/receive antennas, namely $\beta>1$ and $\gamma<1$, the average MMSE degrades. Increasing the amplification level on the relays $(\nu=40 \mathrm{~dB})$ only provides marginal gain. 


\section{CONCLUSION}

In this paper, a large system analysis of DH AF MIMO MAC was considered. We considered the average MMSE which dictates the performance of single-user receivers after multiuser MMSE filtering. A tight lower bound for the average MMSE was proposed based on an eigenvalue-based trace inequality for products of matrices. The bound has been expressed using the a.e.c.d.f. of certain functions of first/second hop channel matrices, which were subsequently calculated using free-probabilistic arguments. Finally, it was shown that in a DH AF MIMO MAC deployment with MMSE filtering the following conditions between first and second hop load should apply, $\beta \leq 1$ and $\beta \gamma \leq 1$, in order to achieve low average MMSE.

\section{REFERENCES}

[1] A. Wyner, "Shannon-theoretic approach to a Gaussian cellular multipleaccess channel," IEEE Trans. Inf. Theory, vol. 40, no. 6, pp. 1713-1727, Nov 1994.

[2] N. Letzepis and A. Grant, "Capacity of the multiple spot beam satellite channel with rician fading," IEEE Trans. Inf. Theory, vol. 54, no. 11, pp. $5210-5222$, nov. 2008 .

[3] V. Morgenshtern and H. Bolcskei, "Crystallization in large wireless networks," IEEE Trans. Inf. Theory, vol. 53, no. 10, pp. $3319-3349$, oct. 2007.

[4] J. Wagner, B. Rankov, and A. Wittneben, "Large n analysis of amplifyand-forward MIMO relay channels with correlated rayleigh fading," IEEE Trans. Inf. Theory, vol. 54, no. 12, pp. 5735 - 5746, Dec 2008.

[5] C.-K. Wen and K.-K. Wong, "On the sum-rate of uplink MIMO cellular systems with amplify-and-forward relaying and collaborative base stations," IEEE J. Select. Areas Commun., vol. 28, no. 9, pp. 1409 -1424 , december 2010.

[6] C. Wen, J. Chen, and P. Ting, "Efficient approach for evaluating throughput of multi-hop MIMO amplify-and-forward relays," Wireless Communications Letters, IEEE, no. 99, pp. 1 -4, 2012.

[7] R. Müller, "A random matrix model of communication via antenna arrays," IEEE Trans. Inf. Theory, vol. 48, no. 9, pp. 2495-2506, Sep 2002.

[8] S.-p. Yeh and O. Leveque, "Asymptotic capacity of multi-level amplifyand-forward relay networks," in Information Theory, 2007. ISIT 2007. IEEE International Symposium on, june 2007, pp. $1436-1440$.

[9] C. Xing, S. Ma, and Y.-C. Wu, "Robust joint design of linear relay precoder and destination equalizer for dual-hop amplify-and-forward MIMO relay systems," Signal Processing, IEEE Transactions on, vol. 58, no. 4, pp. $2273-2283$, april 2010.

[10] S. Chatzinotas, M. Imran, and R. Hoshyar, "Reduced-complexity multicell decoding systems with multiple antennas at the base station," in 5th International Wireless Communications and Mobile Computing Conference (IWCMC'09), Leipzig, Germany, Jun 2009.

[11] W. Guan and H. Luo, "Joint MMSE transceiver design in nonregenerative MIMO relay systems," IEEE Commun. Lett., vol. 12, no. 7, pp. $517-519$, july 2008 .

[12] A. Lozano and A. Tulino, "Capacity of multiple-transmit multiplereceive antenna architectures," IEEE Trans. Inf. Theory, vol. 48, no. 12 pp. $3117-3128$, dec 2002 .

[13] A. M. Tulino and S. Verdú, "Random matrix theory and wireless communications," Commun. Inf. Theory, vol. 1, no. 1, pp. 1-182, 2004.

[14] C. Martin and B. Ottersten, "Asymptotic eigenvalue distributions and capacity for MIMO channels under correlated fading," IEEE Trans. Wireless Commun., vol. 3, no. 4, pp. 1350-1359, Jul 2004.

[15] J. Lasserre, "A trace inequality for matrix product," IEEE Trans. Automat. Contr., vol. 40, no. 8, pp. 1500 -1501, aug 1995.

[16] D. Voiculescu, "Asymptotically commuting finite rank unitary operators without commuting approximants," Acta Sci. Math., vol. 45, pp. 429431, 1983.

[17] F. Hiai and D. Petz, "Asymptotic freeness almost everywhere for random matrices," Acta Sci. Math. (Szeged), vol. 66, pp. 801-826, 2000.

[18] — - "The semicircle law, free random variables and entropy," Mathematical Surveys and Monographs, vol. 77, 2000.

[19] Z. D. Bai, "Methodologies in spectral analysis of large dimensional random matrices, a review," Statistica Sinica, vol. 9, pp. 611-677, 1999.

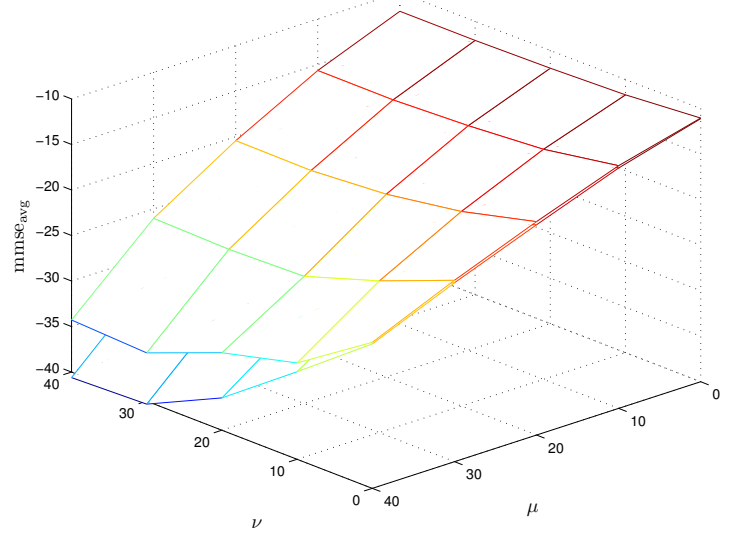

Fig. 1. Average MMSE scaling vs. power levels $\mu, \nu$ in $\mathrm{dBs}$. Parameters $\beta=\gamma=1$. The proposed lower bound is tight for all $\mu, \nu$.

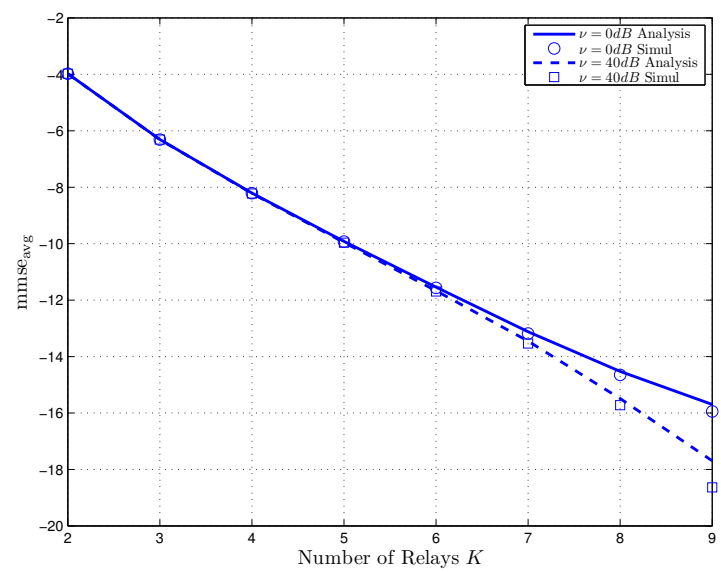

Fig. 2. Average MMSE scaling vs. number of relays $K$. Parameters $M=$ $N=10, \nu=0,40 d B$.

[20] S. Chatzinotas, M. Imran, and C. Tzaras, "On the capacity of variable density cellular systems under multicell decoding," IEEE Commun. Lett., vol. 12, no. 7, pp. 496 - 498, Jul 2008.

[21] S. Chatzinotas, M. Imran, and R. Hoshyar, "On the multicell processing capacity of the cellular MIMO uplink channel in correlated Rayleigh fading environment," IEEE Trans. Wireless Commun., vol. 8, no. 7, pp. 3704-3715, July 2009.

[22] Z. H. Husheng Li and H. Poor, "Asymptotic analysis of large cooperative relay networks using random matrix theory," EURASIP Journal on Advances in Signal Processing, 2008, article ID 235867.

[23] S. Chatzinotas and B. Ottersten, "Free probability based capacity calculation of multiantenna Gaussian fading channels with cochannel interference," Physical Communication, vol. 4, no. 3, pp. $206-$ 217, 2011, Recent Advances in Cooperative Communications for Wireless Systems. [Online]. Available: http://www.sciencedirect.com/ science/article/pii/S1874490711000188

[24] — , "Interference mitigation techniques for clustered multicell joint decoding systems," EURASIP Journal on Wireless Communications and Networking, Multicell Cooperation for Next Generation Communication Systems Series, vol. 132, 2011. [Online]. Available: http://jwen. eurasipjournals.com/content/2011/1/132

[25] N. Letzepis and A. Grant, "Shannon transform of certain matrix products," in Information Theory, 2007. ISIT 2007. IEEE International Symposium on, june 2007, pp. $1646-1650$.

[26] V. Marčenko and L. Pastur, "Distributions of eigenvalues of some sets of random matrices," Math. USSR-Sb., vol. 1, pp. 507-536, 1967.

[27] W. Zhang, G. Abreu, M. Inamori, and Y. Sanada, "Spectrum sensing algorithms via finite random matrices," IEEE Trans. Commun., vol. 60, no. 1 , pp. $164-175$, january 2012. 